\title{
SUMS OF COUNTABLE PRIMARY GROUPS
}

PAUL HILL

This note contains, in particular, a short proof of the celebrated result of $\mathrm{G}$. Kolettis which states that a group $G$ is determined by its Ulm invariants if $G$ is the direct sum of countable, reduced, $p$-primary groups [1]. All groups considered are commutative.

Theorem. Suppose that $G=\sum_{\lambda \in \Lambda} A_{\lambda}$ and $H=\sum_{\lambda \in \Lambda} B_{\lambda}$ are decompositions of $G$ and $H$ into direct sums of countable, reduced, p-groups. If $G$ and $H$ have the same Ulm invariants, then there exists a partition of $\Lambda$ into countable subsets $\Lambda_{\mu}, \mu \in M$, such that $G_{\mu}=\sum_{\lambda \in \Lambda_{\mu}} A_{\lambda}$ is isomorphic to $H_{\mu}=\sum_{\lambda \in \Lambda \mu} B_{\lambda}$ for each $\mu \in M$.

Proof. The theorem is vacuous if $\Lambda$ is countable, so assume that $\Lambda$ is uncountable and let $\Omega$ be the smallest ordinal having the cardinality of the set $\Lambda$. Let $G[p]=\sum_{i \in I}\left\{x_{i}\right\}$. Since $G$ and $H$ are direct sums of countable groups and have the same Ulm invariants, there is ${ }^{1}$ a height-preserving isomorphism $\pi$ from $G[p]$ on to $H[p]$-an element of $G$ is said to have height $\alpha$ if it is contained in $p^{\alpha} G$ but not in $p^{\alpha+1} G$. Thus corresponding to the decomposition $G[p]=\sum_{i \in I}\left\{x_{i}\right\}$ is the decomposition $H[p]=\sum_{i \in I}\left\{y_{i}\right\}$ where $y_{i}=\pi\left(x_{i}\right)$. Since $|G[p]|$ $=|G|=|\Lambda|=|\Omega|$, the index set $I$ can be taken as the initial segment of ordinals less than $\Omega$.

Suppose that $\gamma<\Omega$ and that for each $\beta<\gamma$ we have shown the existence of a subset $S_{B}$ of $\Lambda$ and a subset $I_{B}$ of $I$ such that the following conditions are satisfied.

$$
\begin{gathered}
\sum_{\lambda \in S_{\beta}} A_{\lambda}[p]=\sum_{i \in I_{\beta}}\left\{x_{i}\right\} \text { and } \sum_{\lambda \in S_{\beta}} B_{\lambda}[p]=\sum_{i \in I_{\beta}}\left\{y_{i}\right\} . \\
\left|S_{\beta}\right|=\left|I_{\beta}\right| \leqq \aleph_{0}|\beta| . \\
\alpha \in I_{\beta} \text { if } \alpha<\beta . \\
S_{\beta}=\underset{\alpha<\beta}{\bigcup} S_{\alpha} \text { and } I_{\beta}=\bigcup_{\alpha<\beta} I_{\alpha} \text { if } \beta \text { is a limit ordinal. } \\
S_{\alpha} \subseteq S_{\beta} \text { and } I_{\alpha} \subseteq I_{\beta} \text { if } \alpha<\beta .
\end{gathered}
$$

If $\gamma$ is a limit ordinal, we define $S_{\gamma}=\bigcup_{\alpha<\gamma} S_{\alpha}$ and $I_{\gamma}=\bigcup_{\alpha<\gamma} I_{\alpha}$ and observe that the conditions (1)-(5) remain valid for $\beta \leqq \gamma$.

Received by the editors March 28, 1966.

${ }^{1}$ If $G$ is a countable, reduced, primary group, then $G[p]$ can be decomposed as $G[p]=\Sigma S_{\alpha}$ where the elements of $S_{\alpha}$ have height $\alpha$. The result then easily extends to direct sums of countable groups. 
Suppose that $\gamma$ is not a limit ordinal. There is a minimal subset $S_{\gamma, 1}$ of $\Lambda$ such that $\left\{\sum_{s_{\gamma-1}} A_{\gamma}, x_{\gamma-1}\right\} \subseteq \sum_{s_{\gamma, 1}} A_{\lambda}$ and $\left\{\sum_{s_{\gamma-1}} B_{\lambda}, y_{\gamma-1}\right\}$ $\subseteq \sum s_{\gamma, 1} B_{\lambda}$. There is a minimal subset $I_{\gamma, 1}$ of $I$ such that $\sum s_{\gamma_{\gamma, 1}} A_{\lambda}[p]$ $\subseteq \sum_{I_{\gamma, 1}}\left\{x_{i}\right\}$ and $\sum_{S_{\gamma, 1}} B_{\lambda}[p] \subseteq \sum_{I_{\gamma, 1}}\left\{y_{i}\right\}$. Then there is a minimal set $S_{\gamma, 2}\left(\supseteq S_{\gamma, 1}\right)$ of $\Lambda$ such that $\sum_{I_{\gamma, 1}}\left\{x_{i}\right\} \subseteq \sum_{S_{\gamma, 2}} A_{\lambda}$ and $\sum_{I_{\gamma, 1}}\left\{y_{i}\right\}$ $\subseteq \sum_{S_{\gamma}, 2} B_{\lambda}$. Continuing in this way, we obtain ascending sequences $S_{\gamma, n}$ and $I_{\gamma, n}$ such that

$$
\sum_{S_{\gamma, n}} A_{\lambda}[p] \subseteq \sum_{I_{\gamma, n}}\left\{x_{i}\right\} \subseteq \sum_{S_{\gamma, n+1}} A_{\lambda}[p]
$$

and

$$
\sum_{S_{\gamma, n}} B_{\lambda}[p] \subseteq \sum_{I_{\gamma, n}}\left\{y_{i}\right\} \subseteq \sum_{S_{\gamma, n+1}} B_{\lambda}[p]
$$

Note that $\left|S_{\gamma, n}\right| \leqq \boldsymbol{N}_{0}|\boldsymbol{\gamma}|$ for each positive integer $n$. Set $S_{\gamma}=U S_{\gamma, n}$ and $I_{\gamma}=\bigcup I_{\gamma, n}$. Then conditions (1)-(5) hold for $\beta \leqq \gamma$. Obviously we can use the scheme described above to show the existence of countably infinite sets $S_{1}$ and $I_{1}$ which satisfy conditions (1) and (2) when $\beta=1$; hence there exist, for each $\beta<\Omega$, a subset $S_{B}$ of $\Lambda$ and a subset $I_{\beta}$ of $I$ satisfying conditions (1)-(5). Condition (3) implies that $I=\mathrm{U}_{\beta<\Omega} I_{\beta}$ and condition (1) implies that $\Lambda=\mathrm{U}_{\beta<\Omega} S_{\beta}$.

Define $\Lambda_{0}=S_{1}$ and $\Lambda_{\beta}=S_{\beta+1}-S_{\beta}$ for $1 \leqq \beta<\Omega$. We know that $\sum_{\lambda \in \Lambda_{0}} A_{\lambda}$ and $\sum_{\lambda \in \Lambda_{0}} B_{\lambda}$ have the same Ulm invariants, for (the restriction of) $\pi$ is a height-preserving isomorphism between their socles $\sum_{i \in I_{1}}\left\{x_{i}\right\}$ and $\sum_{i \in I_{1}}\left\{y_{i}\right\}$. We wish to show that $\sum_{\lambda \in \Lambda_{\beta}} A_{\lambda}$ and $\sum_{\lambda \in \Lambda_{\beta}} B_{\lambda}$ have the same Ulm invariants. Since

$$
\sum_{S_{\beta+1}} A_{\lambda}=\sum_{S_{\beta}} A_{\lambda}+\sum_{\Lambda_{\beta}} A_{\lambda}
$$

and

$$
\sum_{S_{\beta+1}} B_{\lambda}=\sum_{S_{\beta}} B_{\lambda}+\sum_{\Lambda_{\beta}} B_{\lambda}
$$

and since the height-preserving isomorphism $\pi$ from $\sum s_{s_{1+1}} A_{\lambda}[p]$ onto $\sum_{s_{\beta+1}} B_{\lambda}[p]$ maps $\sum_{S_{\beta}} A_{\lambda}[p]$ on to $\sum_{s_{\beta}} B_{\lambda}[p]$, the composition mapping $\phi \pi$ is a height-preserving isomorphism from $\sum_{\lambda \in \Lambda_{\beta}} A_{\lambda}[p]$ onto $\sum_{\lambda \in \Lambda_{\beta}} B_{\lambda}[p]$ where $\phi$ is the projection of $\sum_{\mathrm{S}_{\beta+1}} B_{\lambda}$ on to $\sum_{\Lambda_{\beta}} B_{\lambda}$. Thus $\sum_{\lambda \in \Lambda_{\beta}} A_{\lambda}$ and $\sum_{\lambda \in \Lambda_{\beta}} B_{\lambda}$ have the same Ulm invariants.

Since $\left|\Lambda_{\beta}\right|^{\beta} \leqq \boldsymbol{\aleph}_{0}|\beta|<|\Lambda|$ for each $\beta<\Omega$, the theorem now follows by induction on $|\Lambda|$.

\section{REFERENCE}

1. G. Kolettis, Direct sums of countable groups, Duke Math. J. 27 (1960), 111-125. 